\title{
Direct Measurement of Entropic Forces Induced by Rigid Rods
}

\author{
L. Helden, ${ }^{1}$ R. Roth, ${ }^{2,3}$ G. H. Koenderink, ${ }^{4}$ P. Leiderer, ${ }^{1}$ and C. Bechinger ${ }^{1}$ \\ ${ }^{1}$ Physics Department, University of Konstanz, D-78457 Konstanz, Germany \\ ${ }^{2}$ Max-Planck-Institute für Metallforschung, Heisenbergstrasse 1, D-70569 Stuttgart, Germany \\ ${ }^{3}$ ITAP, Univerität Stuttgart, Pfaffenwaldring 57, D-70569 Stuttgart, Germany \\ ${ }^{4}$ Van 't Hoff Laboratory for Physical and Colloid Chemistry, Debye Research Institute, Utrecht University, \\ Padualaan 8, $3584 \mathrm{CH}$ Utrecht, The Netherlands
}

(Received 19 August 2002; published 29 January 2003)

\begin{abstract}
We present the first direct depletion potential measurements for a single colloidal sphere close to a wall in a suspension of rigid colloidal rods. Since all particle interactions are as good as hard-core-like, the depletion potentials are of entirely entropic origin. We developed a density functional theory approach that accurately accounts for this experimental situation. The depletion potentials calculated for different rod number densities are in very good quantitative agreement with the experimental results.
\end{abstract}

PACS numbers: 82.70.Dd, 05.40.-a, 71.15.Mb

Though the entropy of an isolated system is maximized in thermal equilibrium, this does not necessarily apply to the corresponding degree of disorder. For example binary hard-sphere mixtures in equilibrium can form amazingly ordered solid structures for certain size ratios and packing fractions [1-3]. From a microscopic point of view, this crystallization can be understood by considering two large hard spheres suspended in a fluid of smaller spheres. If the distance between the large particles becomes smaller than the diameter of the smaller spheres, the concentration of the latter becomes depleted in the gap and an effective osmotic pressure imbalance causes a net attraction between the large particles. Consequently, this effect has been termed depletion force with the smaller particles often being referred to as depletion agent [4]. Because depletion forces are not restricted to particular length scales, the underlying principle is also applicable to macromolecular and biological systems. Accordingly, such interactions are of general interest for physicists, chemists, and engineers. While there has been tremendous progress regarding the understanding of depletion forces in binary sphere systems [5,6] and colloid-polymer mixtures [7,8], less is known about systems where nonspherical objects such as rods are employed as depletion agent. These particles are particulary interesting for the following reasons: (i) Owing to their orientational degree of freedom, rods with high aspect ratios are much more effective as a depletion agent than spherical objects. Thus only minute volume fractions (typically below 1\%) are needed to induce phase separation in sphere-rod systems [9-11]. (ii) In addition to rod-rich and rod-poor phases, a rich phenomenology with, e.g., lamellar and columnar phases in sphere-rod mixtures has been observed which show strong resemblance to micellar and block-copolymer systems [12]. (iii) Rodlike molecules (e.g., actin filaments or DNA) are important in many biological systems where depletion effects are known as macromolecular crowding [13]. Very recently even depletion induced torque effects have been predicted that might play an important role in orientation dependent reactions of biological macromolecules [14].

In this Letter, we report on the first direct measurements of depletion potentials using rigid rods as the depletion agent. Since electrostatic and dispersion forces were largely screened in our experiments, only hard-body interactions had to be considered and depletion forces of entirely entropic origin could be observed. This is in contrast to recent studies of Lin et al., where highly charged, $\mathrm{fd}$ bacteriophage viruses were employed as the depletion agent $[15,16]$. In those systems additional effects such as the rod flexibility and salt-dependent interactions have to be taken into account. In addition, we have calculated the depletion potential within density functional theory (DFT) for the exact sphere-wall geometry of our system. Theory and experiments are in excellent quantitative agreement, confirming the model character of our system.

Using total internal reflection microscopy (TIRM), we were able to obtain the depletion potential of a single polystyrene (PS) sphere (diameter $\sigma_{\mathrm{PS}}=3.7 \mu \mathrm{m}$ [17]) close to a flat wall as a function of the rod number density $\rho$. The basic idea of TIRM (see $[18,19]$ for detailed reviews) is to collect the scattered light from a spherical micron-sized probe particle fluctuating due to its Brownian motion close to a horizontal planar wall. The particle-wall separation can be obtained from the scattering intensity, when the particle is illuminated by an evanescent light field from below. Using the Boltzmann distribution, a distance-dependent particle-wall interaction potential with a resolution of about $0.3 k_{B} T$ can be deduced from the separation histogram.

As a depletion agent we used colloidal rods consisting of a boehmite $(\gamma-\mathrm{AlOOH})$ core [20] coated with a $4.5 \mathrm{~nm}$ thick silica layer [21]. The average length $L$ and diameter $\sigma$ of the resulting rods as determined by transmission electron microscopy (TEM) are $203 \pm 93 \mathrm{~nm}$ and $18 \pm 3 \mathrm{~nm}$, respectively. A typical TEM picture is shown in Fig. 1. To minimize attractive dispersion forces which could lead to considerable changes in the depletion 


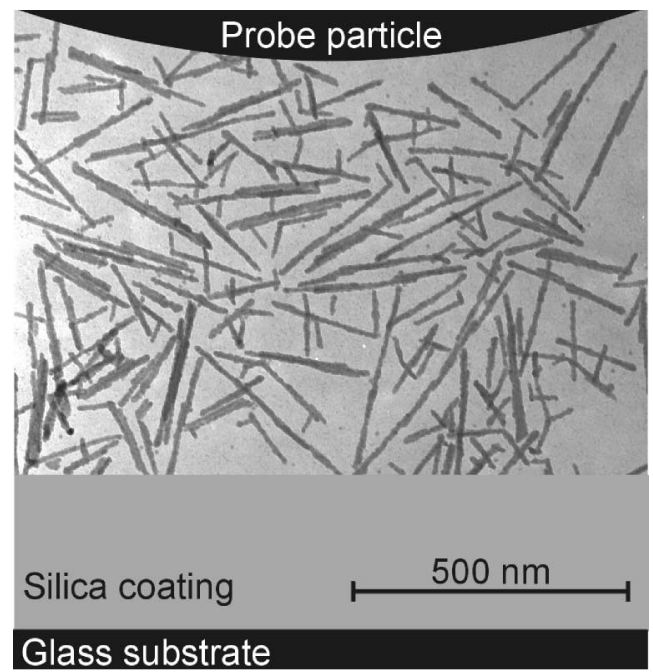

FIG. 1. To scale sketch of the experimental situation including a TEM picture of the rods used as the depletion agent.

interactions [22], dimethylformamide (DMF, refractive index $n=1.43$ ) was used as a solvent. The use of DMF leads to the formation of a solvation layer on the silica surfaces [23] and the dissociation of surface silanol groups. Both effects enhance the stability of the rod suspension. As a result, the suspension remained stable up to at least $0.25 \mathrm{M} \mathrm{LiCl}$. The following measurements were all performed at $0.2 \mathrm{M} \mathrm{LiCl}$ corresponding to a Debye screening length well below $1 \mathrm{~nm}$.

The sample cell comprised two glass microscope slides $(n=1.51)$ which were sealed together with a $0.5 \mathrm{~mm}$ silicon gasket. In order to avoid irreversible sticking of the PS spheres or the rods to the sample cell, the substrate was coated with a $300 \mathrm{~nm}$ thick silica layer $(n \approx 1.45)$ which provides fairly good index matching to the DMF solvent. As a result, interactions of both the rods and the probe particle with the wall can be regarded as hard-core like, as will be demonstrated in detail below. Two thin syringe needles were inserted through the gasket into the sample cell, to control the rod concentration in the cell. The whole cell was optically matched to a glass prism which was illuminated from below with a laser diode $(\lambda=658 \mathrm{~nm})$ to generate an evanescent light field with a characteristic decay length of $207 \mathrm{~nm}$. Other details of the setup are described in [24].

In order to demonstrate the hard-core nature of the interaction potential between the PS sphere and the silica coated glass wall, we first measured interaction potentials without adding rods to the DMF $(0.2 \mathrm{M} \mathrm{LiCl})$ solution. Figure 2, curve $(a)$ shows a typical potential which consists of a long-range linear, attractive gravitational force and a strong, short-ranged repulsion close to the wall. The particle-wall contact $(z=0)$ was determined as the distance where the slopes of the curves exceeded a magnitude of $3 \mathrm{pN}$. This value corresponds to the upper force detection limit of our TIRM setup caused by the $1 \%$

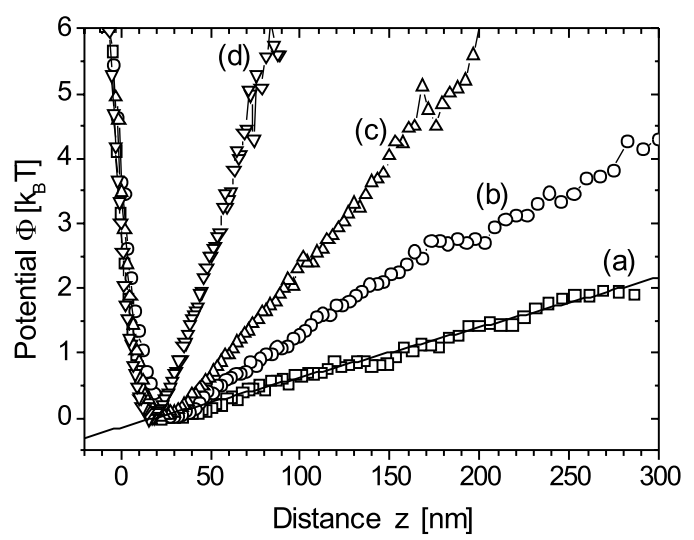

FIG. 2. Particle-wall potentials $\Phi(z)$ of a $3.7 \mu \mathrm{m}$ PS sphere as a function of the particle-wall separation $z$ when no depletion agent is added. While curve $(a)$ is measured on the free probe particle, in curves $(b),(c),(d)$ it experiences an additional vertical light pressure, which increases its effective weight.

rms noise of the laser diode. The particle-wall contact is also in good agreement with calculations based on the distance-dependent diffusion coefficient of the probe particle [25]. The curves $(b)-(d)$ in Fig. 2 were obtained with the same particle but with an additional laser beam (Nd:YAG, $\lambda=532 \mathrm{~nm}$ ) incident from above onto the PS particle. Because of light forces [19] the effective weight of the particle increases with increasing laser power. From the linear slopes of the potential at large distances we determined the effective weight forces to be 37,75 , 147 , and $418 \mathrm{fN}$ for curves $(a)-(d)$, respectively. Note that although the effective weight was increased by more than a factor of 10 in these experiments, the steep repulsive part close to the wall is practically identical for all potentials. Moreover, attractive dispersion forces, which typically can be detected for separations $z<100 \mathrm{~nm}$ [24], were not observed, demonstrating the effectiveness of the silica coating in suppressing these forces. Hence the particle-wall interaction can be considered to a good approximation as hard-core like (in particular on the characteristic length scale of the rods, i.e., $\approx 200 \mathrm{~nm}$ ).

When rods are added to the system, depletion forces modify the effective interaction potential between the PS sphere and the wall. Figure 3 shows the measured potentials (symbols) for different number densities of the rods. The rod number density in the stock dispersion was calculated from the mass fraction of the rods and their number-average size, assuming cylindrical shape. Prior to each measurement, we added rods stock dispersion to the suspension in the sample cell, gently pumped the suspension with the syringe until it was mixed and allowed the system to equilibrate for at least $1 \mathrm{~h}$ before starting the measurements. The rod number densities $\rho_{\text {exp }}$ which are given in the second column of Table I are calculated from the dilution of the stock dispersion. 


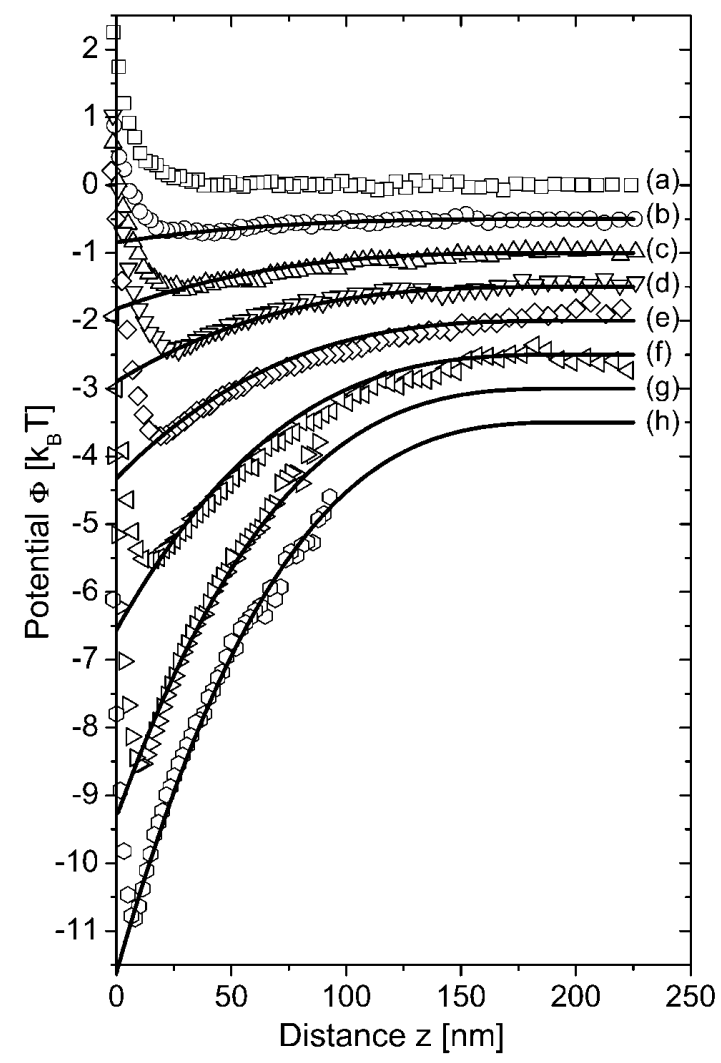

FIG. 3. Measured depletion potentials (symbols) and calculations (lines) for different concentrations of rods $(a)-(h)$ as indicated in Table I. Gravity is subtracted for every curve. For the sake of clarity, all the potentials are shifted by $0.5 k_{B} T$ in the vertical direction.

As can be seen in Fig. 3, with increasing rod concentration the potential minima become deeper and are also slightly shifted closer to the wall. The theoretically expected onset of the depletion forces corresponds to the length $(L)$ of the rods, i.e., $\approx 200 \mathrm{~nm}$. While this onset is difficult to resolve clearly at the smallest rod densities, data for higher densities [Fig. 3, curves $(e),(f)$ ] confirm this theoretical expectation. For the two highest rod con-

TABLE I. Comparison between experimental $\left(\rho_{\text {exp }}\right)$ and calculated $\left(\rho_{\text {DFT }}\right)$ rod number density for each curve of Fig. 3 . The last two columns list the theoretical contact value $[\beta \Phi(z=0)]$ and the depth of the measured potential well $\left(\beta \Phi_{\min }\right)$.

\begin{tabular}{ccccc}
\hline \hline Curve & $\rho_{\exp }\left[\mu \mathrm{m}^{-3}\right]$ & $\rho_{\text {DFT }}\left[\mu \mathrm{m}^{-3}\right]$ & $\beta \Phi(z=0)$ & $\beta \Phi_{\min }$ \\
\hline$(a)$ & 0.0 & 0 & $\ldots$ & $\ldots$ \\
$(b)$ & 4.6 & 4.5 & -0.3 & -0.2 \\
$(c)$ & 9.3 & 10.5 & -0.8 & -0.5 \\
$(d)$ & 21.7 & 18.0 & -1.4 & -1.1 \\
$(e)$ & 34 & 30 & -2.3 & -1.7 \\
$(f)$ & 46 & 52 & -4.0 & -3.0 \\
$(g)$ & 79 & 82 & -6.3 & -5.5 \\
$(h)$ & 103 & 106 & -8.1 & -7.3 \\
\hline \hline
\end{tabular}

centrations [Fig. 3, curves $(g),(h)$ ], depletion effects became so strong that the PS particle was trapped in the potential minimum and did not fluctuate more than $100 \mathrm{~nm}$ away from the surface. Accordingly no statistics could be acquired for larger distances. It is noteworthy that even at the smallest rod number densities $\rho_{\text {exp }}=4.6 \mu \mathrm{m}^{-3}$ used in our experiments the depletion attraction is already visible. If spheres with a diameter equal to the length of the rods would be used at the same volume fraction instead, the maximum depletion attraction at $z=0$ would be less than $4 \%$ of the corresponding rod values [4].

So far several theoretical approaches to describe depletion potentials of a rodlike depletion agent between two spheres or two flat walls have been reported [12,26-29]. To explain our experimental findings we present here the first theory which explicitly takes the sphere-wall geometry of our system into account. It is based on a recently developed DFT for a mixture of hard spheres and ideal needles [30] within the framework of the general approach to depletion potentials $[5,6]$. In this way it is assured that the theory is correct for the given geometry without relying on the validity of the frequently used Derjaguin approximation. A detailed discussion of our model is given elsewhere [31]. We calculated the solid lines in Fig. 3 under the following assumptions: (i) All interactions were modeled as hard-core-like. The system is then fully characterized by the rod aspect ratio $\sigma / L \approx 0.09$, the sphere-rod size ratio $\sigma_{\mathrm{PS}} / L=18.2$, and the number density of the rods $\rho$. (ii) The rods are treated as noninteracting, which dramatically simplifies the model and is a valid assumption for the rod densities used in our experiments. Even the highest experimental density $\left(\rho_{\max }\right)$ is smaller than the density where the average center-to-center distance between the rods is equal to their length $\left(\rho_{L}=L^{-3}=120 \mu \mathrm{m}^{-3}\right)$. Furthermore the pressure of the fluid of rods at $\rho_{\max }$, as, e.g., estimated by a virial expansion up to second order is still described very well by assuming an ideal gas of rods, i.e., $\beta p \approx \rho+\mathcal{O}\left(\rho^{2}\right)$. The isotropic-nematic phase transition occurs at a rod density $\rho^{*}$, over 35 times higher than $\rho_{\max }$ (for the present aspect ratio $\rho^{*} L^{2} \sigma \approx 2.8$ [32]).

The ideal character of the rod fluid results in a simple structure of the depletion potential:

$$
\beta \Phi(z)=-\rho W\left(z, \sigma / L, \sigma_{\mathrm{PS}} / L\right),
$$

where $W\left(z, \sigma / L, \sigma_{\mathrm{PS}} / L\right)$ is, for given ratios $(\sigma / L$, $\left.\sigma_{\mathrm{PS}} / L\right)$, a universal, purely geometric function that has to be calculated numerically. An approximate analytical expression for $W(z)$ in the regime $\sigma_{\mathrm{PS}} / L \gg 1$ can be obtained from first order virial expansion as shown in $[9,27]$. Adopting the sphere-sphere geometry used there to our sphere-wall geometry by multiplying with 2 , $W(z)=\frac{\pi}{6} \frac{\sigma_{\mathrm{PS}}}{L}(L-z)^{3}$ for $z<L$ and $W(z) \equiv 0$ for $z \geq L$. This function very closely resembles our numerical calculations, but yields 5\% higher contact values. 
In order to achieve a quantitative theoretical description of the experiment, we regarded $\rho$ as an adjustable parameter and varied it until best agreement between calculated and measured potentials was obtained. The results are plotted as solid lines in Fig. 3 and show very good agreement with our experimental data. This verifies a posteriori the hard-core nature of the experimental system and that the assumptions implied in Eq. (1) are appropriate. Only for the smallest distances $z<20 \mathrm{~nm}$ in Fig. 3 some deviations between the theory and the experiments can be observed. This is due to remaining repulsive interactions having a finite length scale. The resulting theoretical densities $\rho_{\text {DFT }}$ are compared to the corresponding experimental values $\rho_{\exp }$ in Table I and are in good agreement. The experimental concentrations $\rho_{\text {exp }}$ deviate from $\rho_{\text {DFT }}$ with a standard deviation of only $12 \%$. We attribute this small remaining discrepancy to the uncertainties in preparation and handling of the small $(\approx 0.5 \mathrm{ml})$ amounts of dispersion in the sample cell.

It should be mentioned that the presented DFT approach can easily be extended to higher densities of the depletion agent. This is particularly interesting because in this regime correlation effects become important which are known to cause additional repulsive depletion forces at larger distances [27].

In summary, we have presented the first direct measurement of depletion potentials of a sphere in front of a wall in the presence of rigid hard rods as the depletion agent. The system was prepared in such a way that all interactions could be treated as hard-core-like. We find excellent quantitative agreement between the experimentally determined density dependent depletion potentials and the theoretical calculations based on DFT. Having demonstrated the validity of this theoretical description for ideal rod depletion systems, we hope that our work will stimulate further studies in other fields where rodlike particles or biomolecules are present.

It is a pleasure to thank D. G. A. L. Aarts for the synthesis of the rods and L. Harnau, R. van Roij, A. P. Philipse, H. N.W. Lekkerkerker, and M. Piech for helpful discussions. The financial support of the Deutsche Forschungs Gemeinschaft SFB513 and the Optikzentrum Konstanz are gratefully acknowledged.

[1] P. Bartlett, R. H. Ottewill, and P. N. Pusey, Phys. Rev. Lett. 68, 3801 (1992).

[2] N. Hunt, R. Jardine, and P. Bartlett, Phys. Rev. E 62, 900 (2000).

[3] A. B. Schofield, Phys. Rev. E 64, 51403 (2001).

[4] S. Asakura and F. Oosawa, J. Chem. Phys. 22, 1255 (1954).
[5] B. Götzelmann, R. Roth, S. Dietrich, M. Dijkstra, and R. Evans, Europhys. Lett. 47, 398 (1999).

[6] R. Roth, R. Evans, and S. Dietrich, Phys. Rev. E 62, 5360 (2000).

[7] D. Rudhardt, C. Bechinger, and P. Leiderer, Phys. Rev. Lett. 81, 1330 (1998).

[8] R. Verma, J. C. Crocker, T. C. Lubensky, and A. G. Yodh, Phys. Rev. Lett. 81, 4004 (1998).

[9] G. H. Koenderink, G. A. Vliegenhart, S. G. J. M. Kluijtmans, A. van Blaaderen, A. P. Philipse, and H. N.W. Lekkerkerker, Langmuir 15, 4693 (1999).

[10] G. A. Vliegenthart, A. van Blaaderen, and H. N.W. Lekkerkerker, Faraday Discuss. Chem. Soc. 112, 173 (1999).

[11] G. A. Vliegenthart and H. N.W. Lekkerkerker, J. Chem. Phys. 111, 4153 (1999).

[12] Z. Dogic, D. Frenkel, and S. Fraden, Phys. Rev. E 62, 3925 (2000).

[13] M. Kinoshita and T. Oguni, Chem. Phys. Lett. 351, 79 (2002).

[14] R. Roth, R. van Roij, D. Andrienko, K. R. Mecke, and S. Dietrich, Phys. Rev. Lett. 89, 088301 (2002).

[15] K.-H. Lin, J. C. Crocker, A. C. Zeri, and A. G. Yodh, Phys. Rev. Lett. 87, 088301 (2001).

[16] A. W. C. Lau, K.-H. Lin, and A. G. Yodh, Phys. Rev. E 66, 020401 (2002).

[17] FS05F from Bangs Laboratories, Inc. Fishers, IN. Actual diameter $\sigma_{\mathrm{PS}}=3.69 \pm 0.05 \mu \mathrm{m}$. The particles were cross linked with $11 \%$ divinylbenzene which avoids dissolving in DMF.

[18] J.Y. Walz, Curr. Opin. Colloid Interface Sci. 2, 600 (1997).

[19] D. C. Prieve, Adv. Colloid Interface Sci. 82, 93 (1999).

[20] P. A. Buining, C. Pathmamanoharan, J. B. H. Jansen, and H. N.W. Lekkerkerker, J. Am. Ceram. Soc. 74, 1303 (1991).

[21] M. P. B. van Bruggen, Langmuir 14, 2245 (1998).

[22] C. Bechinger, D. Rudhardt, P. Leiderer, R. Roth, and S. Dietrich, Phys. Rev. Lett. 83, 3960 (1999).

[23] G. H. Koenderink, M. P. Lettinga, and A. P. Phillipse, J. Chem. Phys. 117, 7751 (2002).

[24] H.-H. von Grünberg, L. Helden, P. Leiderer, and C. Bechinger, J. Chem. Phys. 114, 10094 (2001).

[25] M. A. Bevan and D. C. Prieve, J. Chem. Phys. 113, 1228 (2000).

[26] L. Auvray, J. Phys. (Paris) 42, 79 (1981).

[27] Y. Mao, M. E. Cates, and H. N.W. Lekkerkerker, J. Chem. Phys. 106, 3721 (1997).

[28] K. Yaman, C. Jeppesen, and C. M. Marques, Europhys. Lett. 42, 221 (1998).

[29] M. Piech and J. Y. Walz, Langmuir 16, 7895 (2000).

[30] M. Schmidt, Phys. Rev. E 63, 050201 (2001).

[31] R. Roth, J. Phys. Condens. Matter 15, 277-282 (2003).

[32] P. Bolhuis and D. Frenkel, J. Chem. Phys. 106, 666 (1997). 